\section{Sexual health: what's in a name?}

Sonnex et al presented the results of a survey of 150 of their British patients' preference for signposting for their clinic: $65 \%$ chose "clinic 1A", $23 \%$ opted for another euphemistic name-for example, "Lydia clinic", $25 \%$ chose "department of genitourinary medicine" or "GU clinic", $25 \%$ chose "department of sexual health" or "sexual health clinic" and only $8 \%$ chose "genitomedical clinic" (more than one choice was allowed). At that time their clinic apparently operated under the name "clinic $1 \mathrm{~A}-$ genitomedical clinic".

Beginning with the then new Parramatta sexual health clinic in 1979, Australian "STD clinics" have, one by one, adopted the title "sexual health clinic/centre". Our own clinic changed its name in 1990 . The new name was intended to reflect the broader clinical base: which includes family planning/reproductive medicine, sexuality and relationship counselling, and other general aspects of sexual health medicine. The name also implies a proactive population health approach rather than just providing a clinical service. $^{2}$

As there has been little consumer consultation about our name change, we included a question on patients' preference for a clinic name as part of a satisfaction survey.

In all, 563 consecutive general clinic patients completed a satisfaction survey questionnaire in 1996. Exclusion criteria were inability to read English and attendance at a special clinic-for example, HIV eye clinic, colposcopy/gynaecology, Thai, or Chinese clinic. The $M: F$ ratio $(1: 0 \cdot 6)$, mean age (30 years), ratio of new to return patients $(1: 1 \cdot 6)$, and proportion born in Australia $(61 \%)$ were all consistent with the clinic's general patient profile. The patients' (mutually exclusive) responses appear in the table.

The majority of our patients responded favourably or indifferently to the centre's new name. About one in five preferred "Nightingale clinic" (a name that had been promoted in the 1980s) but it was unclear whether this was an expression of preference for a euphemistic name or a desire to commemorate the fact that the building in which the centre is located is the cradle of nursing in Australia. We were surprised at the unpopularity of the names "STD clinic" and "genitourinary medicine clinic" given that the former was the name of the centre 6 years previously and, relevant to the latter name, $15 \%$ of the sample were from the United Kingdom or the Republic of Ireland.

Interpreting Sonnex et al's and our studies together, it appears that patients are relatively accepting of a variety of names for STD/HIV medicine services, particularly the name in current usage. However, "STD clinic" and "genitourinary medicine clinic" have not achieved wide acceptance. The British patients' apparent preoccupation with euphemism seems to contrast with the Australian patients' open minded attitude. It is possible that the broader service profile of Australian sexual health services has helped

Response of patients to a question about their preference for a clinic name $(n=563)$

\begin{tabular}{lr}
\hline Sydney sexual health centre & $40 \cdot 5 \%$ \\
No preference & $32 \cdot 5 \%$ \\
Nightingale clinic & $20 \cdot 2 \%$ \\
STD clinic & $4 \cdot 6 \%$ \\
Genitourinary medicine clinic & $1 \cdot 4 \%$ \\
Other & $0 \cdot 7 \%$ \\
\hline
\end{tabular}

to partially destigmatise them. Alternatively there may be a true cultural difference. Notably, one quarter of the British patients liked "sexual health" despite their lack of previous exposure to the term.

Australia's choice of "sexual health clinic/centre" was driven by a new service philosophy rather than euphemism. We note that most New Zealand clinics and a number of new services in the United Kingdom have also adopted the term. Dissatisfaction with the name "genitourinary medicine" is becoming increasingly explicit. ${ }^{3}$

In accordance with this new name and philosophy for our health services we have developed professional titles: sexual health counsellor (previously health adviser, contact tracer, etc), sexual health nurse, and sexual health physician (previously venereologist, family planning consultant, etc). A vote for a name change from The Australasian College of Venereologists to The Australasian College of Sexual Health Physicians achieved an $84 \%$ approval by those who voted in $1996 .{ }^{4}$ There has also been a well reasoned call for a new specialty of sexual health promotion. ${ }^{5}$

Only time will tell us whether we have found the right name.

ANNETTE DILION BRONWYN ANDERSON BASIL DONOVAN Sydney Sexual Health Centre, Sydney Hospital, PO Box 1614 Sydney, NSW 2001, Australia

1 Sonnex C, Carne CA, Edwards S. Re-thinking sexual health clinics: patients prefer to have
non-descriptive titles (letter). $B M \mathcal{F}$ non-descriptive

2 Donovan B, Neilsen G, Mindel A. Re-thinking sexual health clinics: Australia and New Zealand have taken the lead (letter). $B M^{\mathcal{F}}$ 1995;310:1194.

3 Shahmanesh $M$. On a new road (editorial). Genitourin Med 1996;72:81-2.

4 Moran J. Honorary Secretary's Report. Australasian Sexual Health Physician 1996; 6(3):4-5.

5 Winn M. Sexual health promotion. Venereology, 1996;9:68-73.

Accepted for publication 9 May 1997

\section{Photosensitivity due to saquinavir}

Photosensitivity induced by the HIV proteinase inhibitor saquinavir (Invirase, Roche) is listed as a rare adverse effect in the US Physician's Desk Reference ${ }^{1}$ but is not recorded in the patient information sheet nor in the Summary of Product Characteristics (SmPC) available in the UK. We wish to highlight this discrepancy. A 38 year old man with AIDS (CD4+ count $<10$ cells $\times 10^{6} / 1$ ) and no prior history of photosensitivity underwent 11 sessions of biweekly UVB therapy for a lichenified papular eruption. The UVB dose was chosen according to the patient's skin type and then increased by $40 \%$ every other treatment. Meanwhile because of intolerance of both zidovudine and lamivudine he commenced saquinavir $(1800 \mathrm{mg}$ daily) and stavudine $(60 \mathrm{mg}$ daily), continuing on didanosine $(250 \mathrm{mg}$ daily) and monthly inhaled pentamidine. Three days after starting the new drugs he attended for his twelfth UVB treatment (80 seconds, $40 \%$ UVB) and sustained a severe painful sunburn reaction which settled with topical steroids.

This is the first officially reported case of photosensitivity due to saquinavir. ${ }^{2}$ This adverse reaction is recognised in the USA as reflected by the product labelling and patient information leaflets. ${ }^{3}$ According to Roche's international drug safety expert, the European Medicines Evaluation Agency considered a causal relation unlikely and therefore did not include the risk of photosensitivity in the SmPC for saquinavir. Stavudine induced photosensitivity has not been reported.

Because UVB therapy is useful in managing skin eruptions associated with HIV infection, photosensitivity is an important adverse drug reaction even if it is rare. A San Francisco AIDS Foundation patient information leaflet warns those taking saquinavir to avoid ultraviolet light and use sunblock. ${ }^{3}$ In spite of the current lack of warning in the UK SmPC, we believe patients taking saquinavir should follow the same advice and UVB therapy should be administered warily if at all.

A J WINTER
J M PYWELL
A ILCHYSHYN
J FEARN
D NATIN
Walsgrave Hospitals NHS Trust,
Coventry, CV2 $2 D X$

1 Physicians' Desk Reference. Denver, CO, USA: Computerised Clinical Information System, Micromedex Inc, 1996.

2 Fothergill GA. Drug surveillance manager, Roche, UK. Personal communication 24 February 1997.

3 SFAF leaflet available at http://www.sfaf. org/factsaq.htm

Accepted for publication 13 May 1997

\section{Sexually acquired herpes simplex virus} infection of oropharyngeal cavity

Unprotected orogenital sexual activity has increased in recent years and the reported prevalence varies between $40 \%$ and $70 \% .{ }^{12}$ This altered sexual behaviour could in part be the result of media coverage of the HIV epidemic, which seems to have promoted the belief that orogenital sex is a low risk activity in relation to transmission of HIV and other STDs. Investigations on the effect of such sexual activity on pharyngeal bacterial flora have shown a twofold increase in pharyngeal carriage of Neisseria meningitides in homosexual men ${ }^{1}$ but no increase in asymptomatic carriage or infection with $N$ gonorrhoeae. ${ }^{2}$ However, transmission of $\mathrm{HSV}$ infection from the anogenital region to the oropharyngeal cavity in healthy adults resulting from sexual activity (fellatio and cunnilingus) has not been reported. We report a case of oropharyngeal ulcers due to herpes simplex virus type 1 (HSV-1) infection without associated anogenital herpes, which occurred following orogenital sexual activity (fellatio) in a homosexual male.

A 33 year old homosexual man presented to the genitourinary clinic with a 4 day history of "sore throat", dysphagia and flu-like symptoms and a 2 day history of dysuria and urethral discharge. He reported to have had unprotected, active, and passive orogenital sex (fellatio) with a casual male partner 1 week previously. An examination revealed multiple shallow ulcers with irregular margins, and marked surrounding inflammation on the pharynx, palate, and fauces (fig). There were no lesions on the anogenital region but a copious amount of purulent 